\title{
The Optimum Location for Access Point Deployment based on RSS for Indoor Communication
}

\author{
Oras A. Shareef ${ }^{1}$, Maan M. Abdulwahid ${ }^{2}$, Mahmood F. Mosleh ${ }^{3}$, Raed A. Abd-alhmeed ${ }^{4}$ \\ 1,2,3 Electrical Engineering Techniqal College, Middle Techniqal University, Baghdad, Iraq. \\ ${ }^{4}$ School of Engineering and Informatics, University of Bradford, Bradford, UK. \\ ${ }^{1}$ Oras.a.s.alani@gmail.com; ${ }^{2}$ engmaan.m@gmail.com; ${ }^{3}$ drmahfa@yahoo.com; ${ }^{4}$ r.a.a.abd@bradford.ac.uk.
}

\begin{abstract}
In indoor wireless communication networks, the optimal locations had been known to deploy the access points (AP's) which has a significant impact on improving various aspects of network operation, management, and coverage. In addition, develop the behavioral characteristics of the wireless network. The most used approach for localization purposes was based on Received Signal Strength (RSS) measurements, which is widely used in the wireless network. As well as, it can be easily accessed from different operating systems. In this paper, we proposed an optimal AP localization algorithm based on RSS measurement obtained from different received points. This localization algorithm works as a complementary to the 3D Ray tracing model based REMCOM wireless InSite software and considered two-step localization approach, data collection phase, and localization phase. Obtained result give relatively high accuracy to select the optimum location for AP compare with other selected locations. It is worth to mention that effect of different building materials on signal propagation has been considered with specifying the optimum location of deployment. Furthermore, channel characterizations that based on path losses have been considered as a confirmation for the optimum location being selected.
\end{abstract}

Keywords - mmWave; Wireless InSite; 5G; Localization; propagation.

\section{INTRODUCTION}

Since the past last years, it is agreed that wireless communication has a significant impact on our everyday life [1]. The rapid development of mobile communication, in particular in wireless techniques, have brought new prospects for utilizing the available radio signal [2]. One of the most significant applications for utilizing the radio signal was localization-based services [3, 4], as the wireless signal has been exploited for different localization purposes. Despite satellite which based on localization with including Global Navigation Satellite System (GNSS) has been widely used for outdoor localization environments [5], it cannot be effectively used for the indoor environment. This is because the relative complexity of indoor environment as compared with outdoor one [6], the serious effects of different building material on signal propagation have been proposed [7] with keening to get results with high accuracy, low cost and without needing to provide extra hardware supports.

Indoor localization market had been the witness at rapidly in developing over the recent years with growing in a continuous manner [8]. Locating the Radio Frequency (RF) transmitters or Access Points (AP's) in a wireless network has improved the wide range of purposes [9], including locating the nonfunctional AP, managing and operating the wireless network, as well as estimating the RF propagation characteristics [9].

The development of different approaches and types for accurate and low-cost localization methods, especially for indoors, has recently become one of the most interesting research topics $[9,10,11,12]$. For example, a specific approach has been focused on improving the localization using the most intricate techniques for data collection, where the researcher in [13] improved accuracy by collecting information based on Angle of Arrival (AOA) using a directional antenna. However, such a technique has required extra cost and time [14]. On the other hand, time-based approaches that highlighted in [14] have required time synchronization and additional medium like ultrasound to handle timing issues. Another related approach is $\mathrm{Wi}-\mathrm{Fi}$ based approach which gained higher emphasis by researcher than other approaches, due to the fact that there is no need for additional infrastructure cost. This is due to the fact that most of the buildings are equipped with Wi-Fi AP [15]. The latter approach includes fingerprint based localization or sometimes referred to as RSS based localization, which has been widely investigated by many researchers.

For that, in this paper, the behavior of multipath propagation channel has been investigated at the most common frequency $(2.4 \mathrm{GHz})$ which is used for Wi-Fi networks. In addition, we present AP localization algorithm based on RSS measurement. The later collected from the different received point for each AP location. Additionally, Building material effect for indoor communication has been considered with our localization algorithm, where 3D Ray Tracing simulation approach based REMCOM Wireless InSite software [16] have been utilized in this study. For context, channel characterization based on path loss measurement has been investigated. The rest of the paper is organized as follow, related works, RSS measurement, and localization algorithm is described in section II and III and IV respectively, section V clarified the case study, section VI 
discussed the result and discussion. Finally, section VII presents the conclusion.

\section{RELATED WORKS}

Localization algorithm can be characterized based on two aspects, type of measurements and reference location requirement. The type of measurements is depending on either RSS value or the connectivity between the AP and receivers. The simplest connectivity based-AP localization algorithm is the Centroid algorithm, which has been utilized for Wireless sensor network localization [17]. AP estimating has been achieved by calculating the mean value of the measurement locations, where low accurate results have been obtained by this algorithm. However, connectivity based this method has proposed two assumptions; omnidirectional type of AP antenna and measurements that taken for the entire investigation area [18]. Obtained performance from this method is highly dependent on the measurement data. For the measurement type localization using RSS values, researchers proposed many algorithms for a closed based solution. This includes the Weighted Centroid (WC) algorithm, which has been proposed in [19] for the localization of wireless sensor nodes. Furthermore, WC has been used for wireless AP localization as has been highlighted in [20]. AP location was estimated by calculating weighted function that depends on the RSS measurements. Using this algorithm, the solution for the optimization problem can be determined. However, it uses the LogNormal Shadowing (LNS) model which leads to Nonlinear Least Square problem (NLSP). Consequently, researchers in [21] have presented Linear Least Squares (LLS) algorithm from the development of WC algorithm. LLS has generally used the same formulas of $\mathrm{WC}$, but without estimating the parameters of signal propagation. Additionally, a different Path Loss model was used instead of the LNS model. Even though, this algorithm demonstrated low accuracy in performance. In contrast, researchers in $[22,23]$ have used iterative algorithm represented by Gauss-Newton (GN) and Levenberg-Marquardt (LM) algorithms to overcome the NLSP, which based on differentiation methods. These algorithms showed higher accuracy in performance as than WC algorithms. However, it shows ineffectiveness in several separate area scenarios. New methods have been used by many researchers as in [24] where Importance Sampling algorithm based on Monte Carlo algorithm type was presented. This later algorithm could achieve relatively high accuracy results. However, the expense of computational complexity is high. It is worth to mention that all these previous methods relied on a set of RSS measurements with pre-known reference locations. However, these hypotheses cannot always feasible especially for indoor environments because of the reference location estimation issue.

Many other researchers have started to present new methods and algorithms for AP localization without considering reference locations estimation. The most interesting methods were based on the use of Genetic Algorithm (GA) which has been widely used for AP placement problem $[25,26]$. The researcher in $[25]$ presented the wireless AP placement technique for optimal wireless signal coverage inside buildings. They used the empirical model for indoor wave propagation where path loss depends on the type of walls and floors. In addition, the researcher in [26] present algorithm for optimum AP deployment using Ray-Tracing method for signal propagation and based on the GA algorithm. In contrast, using GA may increase computational complexities.

Inspired by $[25,26]$, our proposed localization algorithm focused on obtaining the optimum location for AP deployment based on RSS measurement. It included twostep for localization with the related database for storing RSS measurements. The effects of different building materials and the building construction on signal propagation were considered with our algorithm. In addition, Ray-Tracing model was proposed for signal propagation and used longdistance model for path loss calculation.

\section{RSS MEASUREMENT}

RSS is a measured value of power transmitted from an $\mathrm{AP}$ at a certain time and received by a wireless device placed in different locations. In addition, it has been considered the most significant approaches in recent localization systems [9], where it works with the noise power to define the Signal to Noise Ratio (SNR) value of the signals. According to Shannon low [27], SNR indicates the valuable capacity of the communication system. In addition to that, RSS measurement constitutes significant role in monitoring AP in Wireless Local Area Network (WLAN) since most of the wireless network provide direct access to the RSS values, as well as its availability in the most operating system such as Android and Microsoft Windows. RSS value represents the received power value with the noise and interference effects as expressed in equation (1) [16]. In addition, RSS measurement is predicted to be used for localization in future millimeter Wave (mmWave) communication systems [28], where the later promises to achieve higher data rate and lower latency communication in the future wireless systems.

$$
R S S=R_{R}+I_{\text {total }}+N_{\text {total }}
$$

where $P_{n}$ is the received power, $I_{\operatorname{tatal}}$ is the total interferences and $\mathbb{V}_{\text {total }}$ is the total noise within the investigated area. Moreover, the result obtained from Wireless InSite represents the received power without considering the effect of noise and interference sources. These results were entered to form a database through the use of a dedicated window designed for this purpose as seen in Fig. 1. 


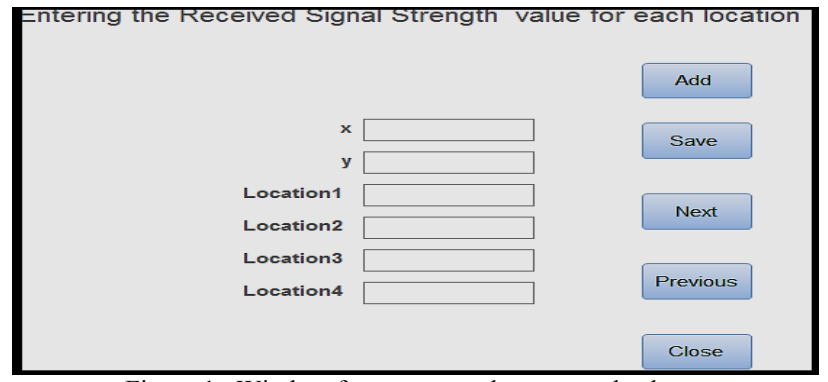

Figure 1. Window form to enter data to our database.

\section{LOCALIZATION ALGORITHM}

Localization algorithm implies on the estimating of different AP or transmitter issue. For our presented algorithm it implies the estimation of optimum AP location for deployment with considering two steps localization approaches, data collection phase, and localization phase. This dedicated algorithm was designed using visualized MATLAB program with a contemporary Graphical User Interface (GUI) window, as shown in Fig. 2. The algorithm works as complementary to the simulation results obtained from wireless InSite software. The received power $\left(P_{R}\right)$ for each fixed received point location is calculated via the later software, basing on equation (2) [16].

$$
R_{n}=\frac{\lambda \beta R_{T}}{(4 \pi)^{2} R^{2}}
$$

where $P_{T}$ is the time-averaged radiated power, $R$ is the separation distance between the transmitter and receiver and $\beta$ is the overlap of the frequency spectrum of the transmitted waveform.

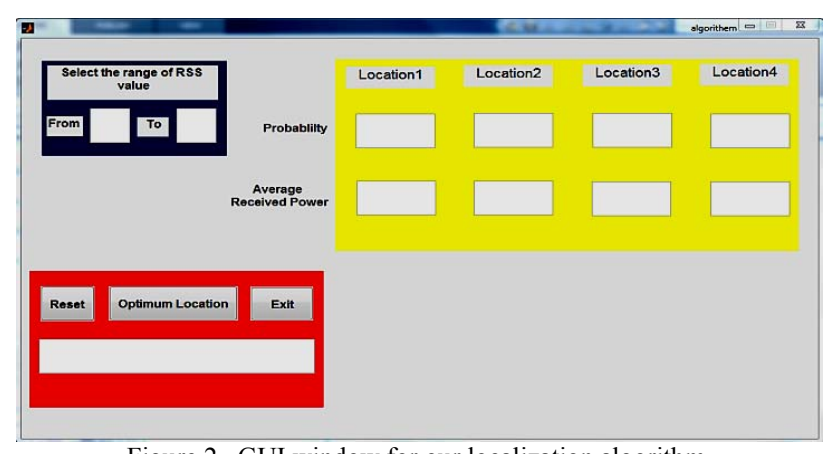

Figure 2. GUI window for our localization algorithm.

Utilising algorithm in this work is considered a set of steps and procedures as expressed in Fig. 3. First phase steps are listed below where:-

Step 1: measuring RSS value obtained from each received points using wireless InSite software.

Step 2: forming a database and collecting RSS values obtained from each received point and for each AP location. For our scenario, it has been investigated from four different locations for AP deployment then the optimum location will be selected among others.

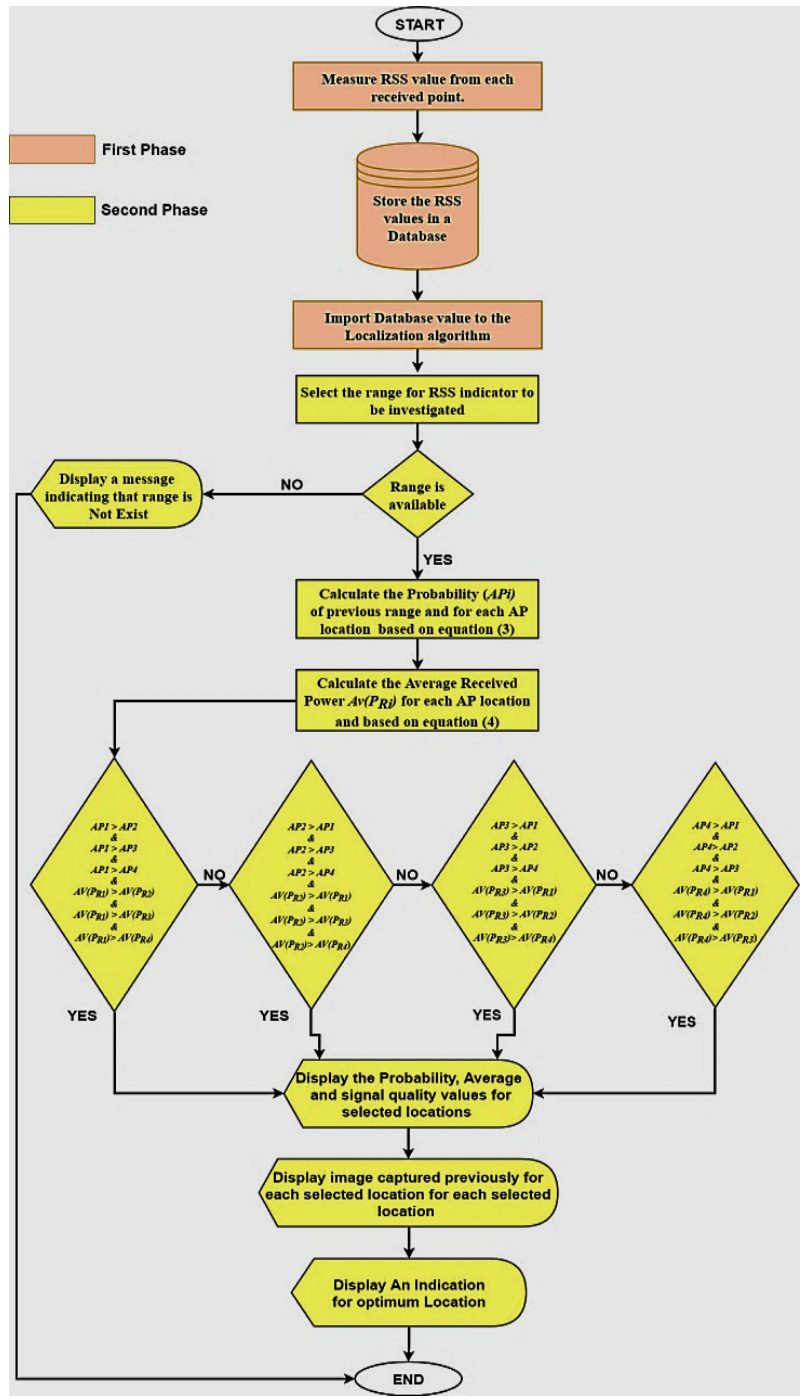

Figure 3. Algorithm flowchart describing its steps and procedures.

Step 3: selecting the value range for RSS value to be incorporated with our algorithm, where the excellent range would be selected as $(>=60 \mathrm{dBm})$ based on many publications such as in [29].

The second phase is achieved by the different type of calculations where:-

Step 1: the probability of the previously selected range of RSS in each AP location would be calculated and based on equation (3) [30].

$$
A P t=\frac{W h}{N_{l}}
$$

where $A P l$ is the probability of AP location and for $i=1: 4$, $\$ M L$ is the $J^{\text {th }}$ location for received points with RSS value (>= $-60 \mathrm{dBm}$ ) and $N_{T}$ is the total number of received points for each AP location where $(J<=T)$.

Step 2: starts by calculating the average received power for each AP location, where the location with the highest 
average can be considered as verification of being the optimum location for deployment AP. Equation (4) [16] is used to calculate the average received power, as follow:

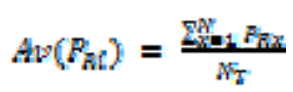

where $A v\left(R_{n c}\right)$ is the average received power for $i^{\text {th }} \mathrm{AP}$ location and $P_{R x}$ is the received power value obtained from each $x$ received point within each AP location.

Step 3: a comparison is made between the probability and average values from each location resulting in displaying the highest value between all AP locations, indicating the optimum location.

\section{CASE STUDY}

The investigated case study was the 2nd floor in the laboratory building of electrical and engineering technical college. Wireless InSite software used to design, load and simulate the second-floor plan as shown in Fig. (4-a), where we investigated the dissemination of four different locations for the AP device to be utilized with our algorithm for optimum location estimation. The height of AP in each of the fourth locations was consolidated with $2.5 \mathrm{~m}$ above the floor ground. For the receiver points, it has been distributed in the middle of every room on the second floor. As well as, a continuous route along the corridor with $4 \mathrm{~m}$ separation distance between each point as shown in Fig. (4-b) with a total number of (54) received point and with the same height of $1.10 \mathrm{~m}$ above the ground. The antenna properties used for both transmitters and receivers described in Table I. The serious effect of different building materials on propagation characteristics was taken into consideration for the entire investigation, where each material thickness, conductivity $(\sigma)$ and relative permittivity $\left(\eta^{\prime}\right)$ was calculated based on the recommendation of International Telecommunication Union (ITU) and listed in Table II. The bandwidth of $20 \mathrm{MHz}$ is selected for our investigation along with the $2.4 \mathrm{GHz}$ band.

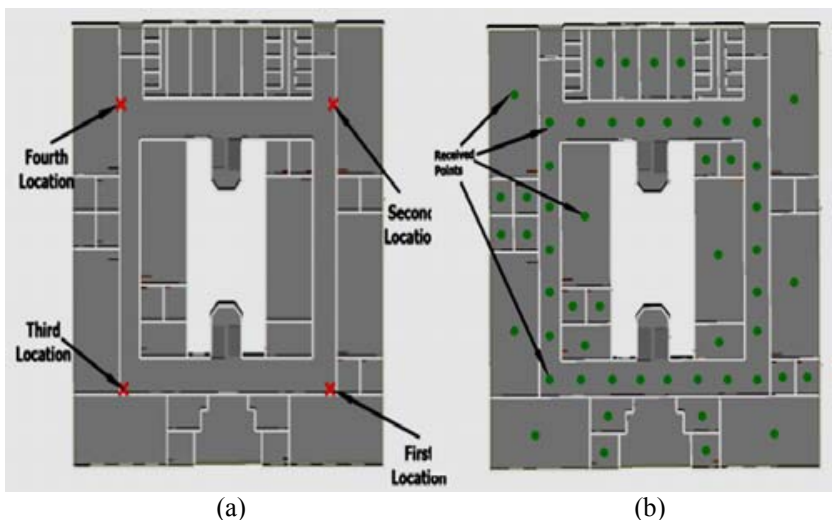

Figure 4. Second-floor layout with (a) selected Access Point locations and (b) received points locations.
TABLE I. TRANSMITTR AND RECEIVER ANTENNA PROPRIETIES

\begin{tabular}{|l|c|c|}
\hline $\begin{array}{l}\text { Antenna } \\
\text { properties }\end{array}$ & Tx Antenna & Rx Antenna \\
\hline Antenna type & Omni-Directional & Omni-Directional \\
\hline Input Power $(\mathrm{dBm})$ & 30 & - \\
\hline Gain $(\mathrm{dBi})$ & 9 & 1.8 \\
\hline E-Plane HPBW & $90^{\circ}$ & $90^{\circ}$ \\
\hline Waveform & Sinusoid & Sinusoid \\
\hline Temperature (k) & 293 & 293 \\
\hline VSWR & 1 & 1 \\
\hline Polarization & $\mathrm{V}$ & $\mathrm{V}$ \\
\hline
\end{tabular}

TABLE II. MATERIAL THICKNESS, CONDUCTIVITY AND PERMITTIVITY VALUES

\begin{tabular}{|l|c|c|c|}
\hline Materials & Thickness (m) & $\boldsymbol{\eta}^{`}$ & $\boldsymbol{\sigma}$ \\
\hline Concrete & 30 & 5.31 & 0.066 \\
\hline Wood & 4.5 & 1.99 & 0.012 \\
\hline Glass & 0.3 & 6.27 & 0.012 \\
\hline Brick & 28 & 3.75 & 0.038 \\
\hline Ceiling Board & 0.9 & 3.66 & 0.001 \\
\hline Floor Board & 2.2 & 1.5 & 0.014 \\
\hline
\end{tabular}

\section{RESULTS AND DISCUSSION}

Wireless signal penetrated in all direction and reflects off walls and other objects. As a result of reflection, multiple copies of the original signal with different delay and attenuation arrived at the received points. This phenomenon is known as multipath. Multipath propagation characteristics based on path losses was considered with the investigation as a confirmation for optimum AP location obtained from our localization algorithm described previously. Fig. 5 Illustrates and evaluates the performance of received power level versus the separation distance between the AP to every received point in the four selected AP locations. The overall trends show significant variation in recorded results obtained from four AP locations due to reasons related to the reflection of different obstacles and effects of different materials on signal propagation. As a result of that, it can be noticed that AP in the fourth location recorded the best overall values as compared to the other three selected locations. On the other hand, when applying our localization algorithm on the four location cases with the appropriate range of RSS values, probability result has shown values of $(0.68,0.64,0.72$ and 0.74$)$ for first, second, third and fourth location respectively. In addition, the value of average received power were $(-49,-58,-46$ and -45$)$ for the same locations respectively. Hence, indicating the optimal location for the access point to achieve the best coverage inside the building floor, which in our scenario was the fourth location. 


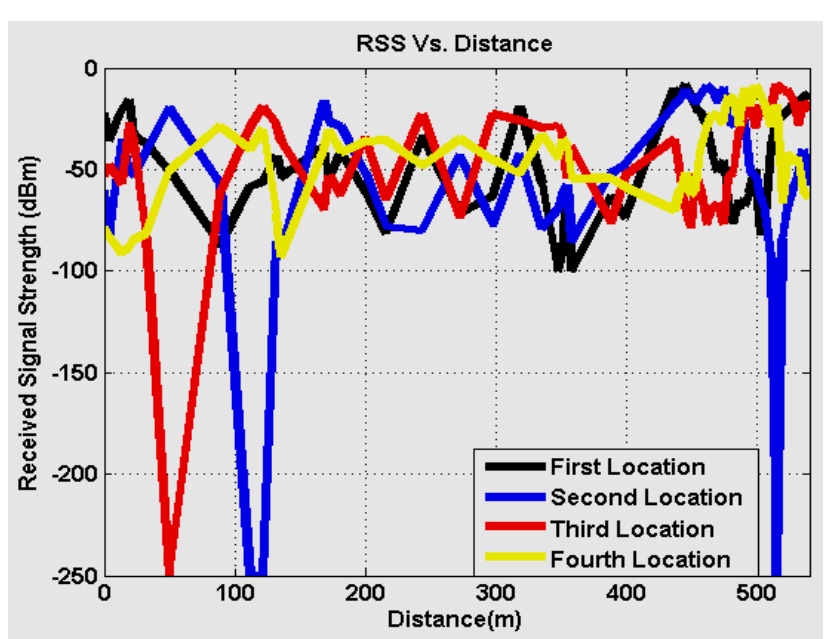

Figure 5. Received power vs. separation distance for four AP.

Finally, to display the relationship between path loss versus the separation distance, Fig. 6 illustrates the relationship in four AP locations. Generally, increasing in separation distance leads to raise the path loss, noted that the relation is not shown in a consistent manner, due to many reasons related with received points being located in the middle of every room within the second floor which increase the possibility of exposure to obstacles and barriers, for example, walls, doors, glasses, and other objects. Additionally, in order to obtain a brief overview, the average value of path loss in the four AP locations was calculated, the values were $(89,97,88$ and 88$) \mathrm{dB}$ for first, second, third and fourth location respectively. The last values denoted that third and fourth locations recorded the approximately same average value for path loss. This is an indication that they are the best locations with fewer losses as compared to the rest.

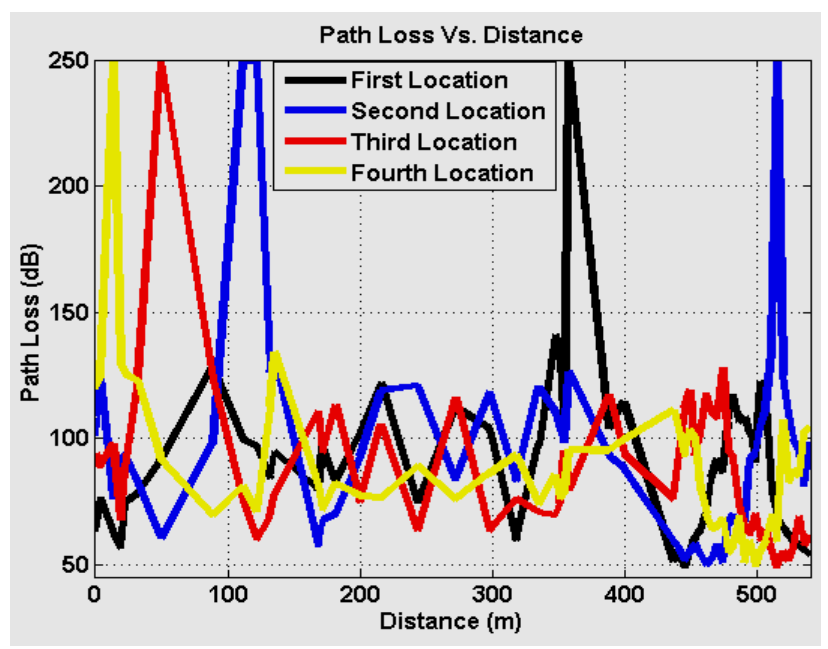

Figure 6. Path loss vs. separation distance for four AP locations.

\section{CONCLUSION}

This paper presents the localization algorithm for the deployment of AP's based on the simulation measurement of RSS. Measurement results obtained from Wireless InSite software and from every received point located in the same fixed location within each AP location. Our presented algorithm works as a complemented part for the accurate results obtained from the later software. In addition, the algorithm focused on the two-step localization approach, including the data collection phase and the localization phase. For the first phase, a dedicated software was designed to collect data in a database to be processed by the second phase. Four AP locations have been selected to be investigated for the estimation of the optimum location dissemination. In this paper, algorithm results show that AP in the fourth location was the optimum location for network deployment, The probability of acceptable selected RSS range per this location was $(0.74)$. Moreover, the previous location recorded the highest mean of received power value as compared to other location results, which it was (-45) $\mathrm{dBm}$. In addition to that, the channel characterized based on path loss has been examined, where the optimal selected location has been confirmed to have the smallest path loss values as compared to other locations. In the future work, proposed a localization algorithm that concluded from this paper can be used to perform experimental works on the real environments and may compare these measurements with the simulation results that obtained from this work. Furthermore, using the presented algorithm and method may contribute to enhance many aspects for localization applications and location estimation issues with lower cost and time for computational procedures.

\section{REFERENCES}

[1] A. Osseiran, V. Braun, T. Hidekazu, P. Marsch, H. Schotten, H. Tullberg, M. A. Uusitalo, and M. Schellman, "The foundation of the mobile and wireless communications system for 2020 and beyond: Challenges, enablers and technology solutions," in Vehicular Technology Conference (VTC Spring), 2013 IEEE 77th. IEEE, 2013, pp. 1-5.

[2] P. Jiang, Y. Zhang, W. Fu, H. Liu, and X. Su, "Indoor mobile localization based on Wi-Fi fingerprint's important access point," International Journal of Distributed Sensor Networks, vol. 11, no. 4,p. 429104, 2015.

[3] I. F. Akyildiz, W. Su, Y. Sankarasubramaniam, and E. Cayirci, "A survey on sensor networks," IEEE communications magazine, vol. 40 , no. 8, pp. $102-114,2002$.

[4] L. Popov et al., "inav: A hybrid approach to wifi localization and tracking of mobile devices," Ph.D. dissertation, Massachusetts Institute of Technology, 2008..

[5] J. Talvitie, "Algorithms and methods for received signal strength based wireless localization," Tampereen teknillinen yliopisto. Julkaisu-Tampere University of Technology. Publication; 1365, 2016

[6] C. Feng, W. S. A. Au, S. Valaee, and Z. Tan, "Received-signalstrength-based indoor positioning using compressive sensing," IEEE Transactions on Mobile Computing, vol. 11, no. 12, pp. 1983-1993, 2012 . 
[7] X. Zhu and Y. Feng, "Rssi-based algorithm for indoor localization," Communications and Network,vol. 5, no. 02, p. 37, 2013.

[8] PR Newswire Europe via COMTEX "Indoor Location Market Worth 40.99 Billion USD by 2022." [Online]. Available: https://www.marketwatch.com/press-release/indoor-location-marketworth-4099-billion-usd-by-2022-2017-10-19-10203318, Oct 19, 2017, Accessed Jan. 9, 2019.

[9] H. Nurminen, M. Dashti, and R. Pich'e, "A survey on wireless transmitter localization using signal strength measurements," Wireless Communications and Mobile Computing, vol. 2017, 2017.

[10] M. A. Al-Ammar, S. Alhadhrami, A. Al-Salman, A. Alarifi, H. S. AlKhalifa, A. Alnafessah, and M. Alsaleh, "Comparative survey of indoor positioning technologies, techniques, and algorithms," in Cyberworlds (CW), 2014 International Conference on. IEEE, 2014, pp. 245-252.

[11] D. Dardari, P. Closas, and P. M. Djuric, "Indoor tracking: Theory, methods, and technologies." IEEE Trans. Vehicular Technology, vol. 64, no. 4, pp. 1263-1278, 2015.

[12] Z. Farid, R. Nordin, and M. Ismail, "Recent advances in wireless indoor localization techniques and system," Journal of Computer Networks and Communications, vol. 2013, 2013.

[13] A. P. Subramanian, P. Deshpande, J. Gao, and S. R. Das, "Drive-by localization of roadside wifi networks, in INFOCOM 2008. The $27^{\text {th }}$ Conference on Computer Communications. IEEE. IEEE, 2008, pp. 718-725.

[14] S. Aditya, A. F. Molisch, and H. M. Behairy, "A survey on the impact of multipath on wideband time-of arrival-based localization," Proceedings of the IEEE, no. 99, pp. 1-21, 2018.

[15] T. Hirahara, T. Yamanoue, H. Anzai, and I. Arita, "Sending an image to a large number of nodes in short time using tcp," in Multimedia and Expo, 2000. ICME 2000. 2000 IEEE International Conference on, vol. 2. IEEE, 2000, pp. 987-990.

[16] REMCOM Inc., " The Wireless InSite users manual." version 2.6.3, romcom inc., $315 \mathrm{~s}$. allen st., suite 416 state college, pa16801, November 2012, Jan. 2009

[17] H. Dang and C. Sunta, "Extrapolation of thorium metabolism from beagles to humans," Health physics, vol. 58, no. 1, pp. 107-109, 1990.

[18] D. Han, D. G. Andersen, M. Kaminsky, K. Papagiannaki, and S. Seshan, "Access point localization using local signal strength gradient," in International Conference on Passive and active network measurement. Springer, 2009, pp. 99-108.

[19] J. Blumenthal, R. Grossmann, F. Golatowski, and D. Timmermann, "Weighted centroid localization in zigbee-based sensor networks," in Intelligent Signal Processing, 2007. WISP 2007. IEEE International Symposium on. IEEE, 2007, pp. 1-6.

[20] C. Laurendeau and M. Barbeau, "Centroid localization of uncooperative nodes in wireless networks using a relative span weighting method," EURASIP Journal on Wireless Communications and Networking, vol. 2010, no. 1, p. 567040, 2009.

[21] J. Koo and H. Cha, "Localizing wifi access points using signal strength," IEEE Communications letters, vol. 15, no. 2, pp. 187-189, 2011.

[22] Y. Zhuang, Z. Syed, J. Georgy, and N. El-Sheimy, "Autonomous smartphone-based wifi positioning system by using access points localization and crowdsourcing," Pervasive and Mobile Computing, vol. 18, pp. 118-136, 2015.

[23] M. A. A. Rahman, M. Dashti, and J. Zhang, "Localization of unknown indoor wireless transmitter," in Localization and GNSS (ICL-GNSS), 2013 International Conference on. IEEE, 2013, pp. 16.

[24] M. Kim, J. J. Fielding, and D. Kotz, "Risks of using ap locations discovered through war driving," in International Conference on Pervasive Computing. Springer, 2006, pp. 67-82.

[25] L. Nagy and L. Farkas, "Indoor base station location optimization using genetic algorithms," in Personal, Indoor and Mobile Radio Communications, 2000. PIMRC 2000. The 11th IEEE International Symposium on, vol. 2. IEEE, 2000, pp. 843-846.
[26] T. H. Hussain and A. A. Salman, "Indoor access point optimization using differential evolution," in Proceedings of the Second Kuwait Conference on e-Services and e-Systems. ACM, 2011, p. 22.

[27] C. E. Shannon, "A mathematical theory of communication," Bell system technical journal, vol. 27, no. 3, pp. 379-423, 1948.

[28] F. Lemic, J. Martin, C. Yarp, D. Chan, V. Handziski, R. Brodersen, G. Fettweis, A. Wolisz, and J. Wawrzynek, "Localization as a feature of mmwave communication," in Wireless Communications and Mobile Computing Conference (IWCMC), 2016 International. IEEE, 2016, pp. 1033-1038.

[29] Jan Pedro Tumusok, Jorunn D. Newth "Wi-Fi Signal Strength: What Is a Good Signal And How Do You Measure It." [Online]. Available: https://eyesaas.com/wi-fi-signal-strength/, Accessed: Jan. 11,2019.

[30] Probability Formula "Probability Formula." [Online]. Available: http://www .probabilityformula.org/calculating-probabilityformula.html, Accessed: Jan. 21, 2019. 